\title{
A Practical Method for Analytical Evaluation of Approximate So- lutions of Fisher's Equations
}

\author{
Necdet BiLDiK ${ }^{1, \star}$ and Sinan DENiZ1, ${ }^{1, \star}$ \\ ${ }^{1}$ Department of Mathematics, Faculty of Art and Sciences, Manisa Celal Bayar University, 45140 Manisa, \\ Turkey.
}

\begin{abstract}
In this article, a framework is developed to get more approximate solutions to nonlinear partial differential equations by applying perturbation iteration technique. This technique is modified and improved to solve nonlinear diffusion equations of the Fisher type. Some problems are investigated to illustrate the efficiency of the method. Comparisons between the new results and the solutions obtained by other techniques prove that this technique is highly effective and accurate in solving nonlinear problems. Convergence analysis and error estimate are also provided by using some related theorems. The basic ideas indicated in this work are anticipated to be further developed to handle nonlinear models.
\end{abstract}

\section{Introduction}

In the past few decades many analytical and numerical methods have been suggested to examine the solutions of partial differential equations (PDEs) such as Adomian decomposition method [1, $2]$, variational iteration method[3,9], homotopy perturbation method [10-12], homotopy analysis method [13-16] and others [4-8]. However, even these methods may not always be sufficient to reach satisfactory results. For this reason, much attention has been recently devoted to the newly developed techniques for obtaining more accurate and efficient solutions. Two of these analytical methods are the optimal homotopy asymptotic method and optimal perturbation iteration method which are recently developed by Marinca et al and Deniz et al respectively. Many authors have applied these methods to solve nonlinear differential equations arising in scientific problems [17-22].

In this study, we consider the generalized Fisher's equation

$$
u_{t}=a u_{x x}+b u\left(1-u^{\alpha}\right)
$$

where $a, b$ and $\alpha$ are real numbers. It is used as a model equation for the evolution of a neutron population in a nuclear reactor and a prototype model for a propagating flame. It also represents the propagation of a viral mutant in an infinitely long habitat [23, 24].

A traveling wave solution $u(x, t)=u(\xi=x-c t)$, spreading with a speed $c$, must be positive and bounded [25]. Therefore the boundary conditions for the traveling wave solution are usually

$$
u(\xi \rightarrow-\infty) \rightarrow 1, u(\xi \rightarrow \infty) \rightarrow 0
$$

\footnotetext{
${ }^{\star}$ e-mail: n.bildik@cbu.edu.tr

${ }^{\star}$ e-mail: sinandeniz01@gmail.com
} 
or

$$
u(x, 0)=v(x), \lim _{|x| \rightarrow 1} u(x, t)=0,|x|<1, t>0
$$

where $|x|<1, t>0$ and $v(x)$ is a given function.

In this study, we modify perturbation iteration method (PIM), which is recently constructed by Pakdemirli et al. [26, 27], to apply it to solve PDEs, particularly for the Eq. (1). We also provide the convergence analysis and error estimate of PIM.

\section{Method of Solution}

To give the basic concepts of PIM, let us consider the Fisher's equation in closed form:

$$
F\left(u_{x x}, u_{t}, u\right)=u_{t}+b u^{\alpha+1}-a u_{x x}-b u=0
$$

where $u=u(x, t)$. Introducing the perturbation parameter $\varepsilon=1$ with nonlinear term yields

$$
F\left(u_{x x}, u_{t}, u, \varepsilon\right)=u_{t}+\varepsilon\left(-a u_{x x}-b u+b u^{\alpha+1}\right)=0 .
$$

$F$ can also be divided as

$$
F=S+R
$$

where

$$
S=u_{t}
$$

is the linear simpler part, which is easy to deal with and

$$
R\left(u_{x x}, u, \varepsilon\right)=\varepsilon\left(-a u_{x x}-b u+b u^{\alpha+1}\right)
$$

is the remaining part. One correction term $\left(u_{c}\right)$ is taken from the perturbation expansion as:

$$
u_{n+1}=u_{n}+\varepsilon\left(u_{c}\right)_{n}
$$

where $n \in \mathrm{N} \cup\{0\}$. Perturbation iteration algorithms will be classified with respect to the degrees of derivatives in the Taylor expansions $(m)$. Briefly, this process will be represented as PIA- $m$.

\section{PIA-1:}

Substituting (7) into the remaining part $R$ in (6), then expanding in a Taylor series only with first derivative gives PIA-1:

$$
R+R_{u_{x x}}\left(\left(u_{c}\right)_{n}\right)_{x x} \varepsilon+R_{u}\left(u_{c}\right)_{n} \varepsilon+R_{\varepsilon} \varepsilon=-S
$$

where $R_{u}=\frac{\partial R}{\partial u}, R_{\varepsilon}=\frac{\partial R}{\partial \varepsilon}, R_{u_{x x}}=\frac{\partial R}{\partial u_{x x}}$. Evaluating all derivatives and functions at $\varepsilon=0$ yields

$$
\left(\left(u_{c}\right)_{n}\right)_{t}=a\left(u_{n}\right)_{x x}+b\left(u_{n}\right)-\left(u_{n}\right)_{t}-b\left(u_{n}\right)^{\alpha+1} .
$$

We can easily obtain first correction term $\left(u_{c}\right)_{0}$ from (9) by determining a trial function $u_{0}$ which satisfies initial condition(s). Then, $u_{1}$ is determined by using $\left(u_{c}\right)_{0},(7)$ and initial condition(s). One can obtain satisfactory results by repeating iteration process with the aid of (7) and (9).

\section{PIA-2:}


Following the same procedure as in the previous algorithm with second derivative gives PIA-2:

$$
\begin{aligned}
& R+R_{u_{x x}}\left(\left(u_{c}\right)_{n}\right)_{x x} \varepsilon+R_{u}\left(u_{c}\right)_{n} \varepsilon+R_{\varepsilon} \varepsilon+\frac{\varepsilon^{2} R_{\varepsilon \varepsilon}}{2}+\frac{\varepsilon^{2} R_{u_{x x} u_{x x}}}{2}\left(\left(u_{c}\right)_{n}\right)_{x x}^{2} \\
& +\frac{\varepsilon^{2} R_{u u}}{2}\left(\left(u_{c}\right)_{n}\right)^{2}+\varepsilon^{2} R_{u_{x x} u}\left(\left(u_{c}\right)_{n}\right)_{x x}\left(u_{c}\right)_{n}+\varepsilon^{2} R_{u_{x x} \varepsilon}\left(\left(u_{c}\right)_{n}\right)_{x x}+\varepsilon^{2} R_{u \varepsilon}\left(u_{c}\right)_{n}=-S
\end{aligned}
$$

By computing all derivatives and functions at $\varepsilon=0$, we have

$$
\left(\left(u_{c}\right)_{n}\right)_{t}+(\alpha+1) b\left(u_{n}\right)^{\alpha}\left(u_{c}\right)_{n}=a\left(u_{n}\right)_{x x}+b\left(u_{n}\right)-\left(u_{n}\right)_{t}-b\left(u_{n}\right)^{\alpha+1}
$$

for the Fisher's equation (5). By means of (7) and (11), iterative scheme is developed.

After obtaining first correction term $\left(u_{c}\right)_{0}$, first approximate solutions are defined by using prescribed initial conditions. Suppose now that

$$
\begin{aligned}
& u_{0}=C_{0} \\
& \left(u_{c}\right)_{n}=C_{n+1} .
\end{aligned}
$$

Then we have

$$
\begin{aligned}
& u_{0}=C_{0} \\
& u_{1}=u_{0}+\left(u_{c}\right)_{0}=C_{0}+C_{1} \\
& u_{2}=u_{1}+\left(u_{c}\right)_{1}=C_{0}+C_{1}+C_{2} \\
& u_{3}=u_{2}+\left(u_{c}\right)_{2}=C_{0}+C_{1}+C_{2}+C_{3} \\
& \quad \vdots \\
& u_{n+1}=u_{n}+\left(u_{c}\right)_{n}=C_{0}+C_{1}+C_{2}+\cdots+C_{n+1}=\sum_{i=0}^{n+1} C_{i}
\end{aligned}
$$

Consequently, one can represent the solution as

$$
u(x, t)=\lim _{n \rightarrow \infty} u_{n+1}(x, t)=\sum_{i=0}^{\infty} C_{i} .
$$

\section{Convergence Analysis and Error Estimate}

In this section, we investigate the convergence of the PIM by giving some theorems.

Theorem 1:(Banach's fixed point theorem). Suppose that B be a Banach space and

$$
A: B \rightarrow B
$$

is a nonlinear mapping, and assume that

$$
\|A[u]-A[\bar{u}]\| \leq \beta\|u-\bar{u}\|, u, \bar{u} \in B,
$$

for some constant $\beta<1$. Then A has a unique fixed point. Also, the sequence

$$
u_{n+1}=A\left[u_{n}\right] \text {, }
$$

with an arbitrary choice of $u_{0} \in B$, converges to the fixed point of $A$ and

$$
\left\|u_{r}-u_{s}\right\| \leq\left\|u_{1}-u_{0}\right\| \sum_{j=s-1}^{r-2} \beta^{j} .
$$


The following theorem, which is needed for our analysis, can be deduced from the Banach fixed point theorem [28].

Theorem 2. Let B be a Banach space denoted with a suitable norm $\|$.$\| over which the series \sum_{i=0}^{\infty} C_{i}$ is defined and assume that the initial guess $u_{0}=C_{0}$ remains inside the ball of the solution $u$. The series solution $\sum_{i=0}^{\infty} C_{i}$ converges if there exists $\beta$ such that

$$
\left\|C_{n+1}\right\| \leq \beta\left\|C_{n}\right\|
$$

Proof: We first define a sequence as:

$$
\begin{aligned}
A_{0} & =C_{0} \\
A_{1} & =C_{0}+C_{1} \\
A_{2} & =C_{0}+C_{1}+C_{2} \\
& \vdots \\
A_{n} & =C_{0}+C_{1}+C_{2}+\cdots+C_{n}
\end{aligned}
$$

Now, we must show that $\left\{A_{n}\right\}_{n=0}^{\infty}$ is a Cauchy sequence in $B$. To do this, consider that

$$
\left\|A_{n+1}-A_{n}\right\|=\left\|C_{n+1}\right\| \leq \beta\left\|C_{n}\right\| \leq \beta^{2}\left\|C_{n-1}\right\| \leq \cdots \leq \beta^{n+1}\left\|C_{0}\right\| .
$$

For every $n, k \in \mathrm{N}, n \geq k$ :

$$
\begin{aligned}
& \left\|A_{n}-A_{k}\right\|=\left\|\left(A_{n}-A_{n-1}\right)+\left(A_{n-1}-A_{n-2}\right)+\cdots+\left(A_{k+1}-A_{k}\right)\right\| \\
& \leq\left\|A_{n}-A_{n-1}\right\|+\left\|A_{n-1}-A_{n-2}\right\|+\cdots+\left\|A_{k+1}-A_{k}\right\| \\
& \leq \beta^{n}\left\|C_{0}\right\|+\beta^{n-1}\left\|C_{0}\right\|+\cdots+\beta^{k+1}\left\|C_{0}\right\|=\frac{1-\beta^{n-k}}{1-\beta} \beta^{k+1}\left\|C_{0}\right\|
\end{aligned}
$$

Since we also have $0<\beta<1$, we can obtain from (22)

$$
\lim _{n, k \rightarrow \infty}\left\|A_{n}-A_{k}\right\|=0
$$

Thus, $\left\{A_{n}\right\}_{n=0}^{\infty}$ is a Cauchy sequence in $B$ and this implies that the series solution (14) is convergent.

Theorem 3. If the initial guess $u_{0}=C_{0}$ remains inside the ball of the solution $u$ then $A_{n}=\sum_{i=0}^{n} C_{i}$ also remains inside the ball of the solution.

Proof: Suppose that

$$
C_{0} \in B_{r}(u)
$$

where

$$
B_{r}(u)=\{C \in A \mid\|u-C\|<r\} .
$$

is the ball of the solution $u$. By hypothesis $u=\lim _{n \rightarrow \infty} A_{n}=\sum_{i=0}^{\infty} C_{i}$ and from Theorem 2, we have

$$
\left\|u-A_{n}\right\| \leq \beta^{n+1}\left\|C_{0}\right\|<\left\|C_{0}\right\|<r
$$

where $\beta \in(0,1)$ and $n \in \mathrm{N}$. This completes the proof.

Theorem 4. Assume that the obtained solution $\sum_{i=0}^{\infty} C_{i}$ is convergent to the solution $u$. If the truncated series $\sum_{i=0}^{k} C_{i}$ is used as an approximation to the solution $u$ of problem (4), then the maximum error is given as, 


$$
E_{k} \leq \frac{\beta^{k+1}}{1-\beta}\left\|C_{0}\right\|
$$

Proof: From the Eq. (22), we have

$$
\left\|A_{n}-A_{k}\right\| \leq \frac{1-\beta^{n-k}}{1-\beta} \beta^{k+1}\left\|C_{0}\right\|
$$

for $n \geq k$. By knowing

$$
u=\lim _{n \rightarrow \infty} A_{n}=\sum_{i=0}^{\infty} C_{i}
$$

it is obvious that

$$
\left\|u-\sum_{i=0}^{k} C_{i}\right\| \leq \frac{1-\beta^{n-k}}{1-\beta} \beta^{k+1}\left\|C_{0}\right\|
$$

and also we can write

$$
E_{k}=\left\|u-\sum_{i=0}^{k} C_{i}\right\| \leq \frac{\beta^{k+1}}{1-\beta}\left\|C_{0}\right\|
$$

since $1-\beta^{n-k}<1$. Here $\beta$ is selected as $\beta=\max \left\{\beta_{i}, i=0,1, \ldots, n\right\}$ where

$$
\beta_{i}=\frac{\left\|C_{n+1}\right\|}{\left\|C_{n}\right\|} .
$$

\section{Applications}

In this section, we will take a few examples with a known exact and approximate solutions obtained via other methods in order to show the effectiveness of both perturbation iteration algorithms.

Example 1: Consider the nonlinear Fisher's problem [29, 30]

$$
u_{t}=u_{x x}+u(1-u)
$$

subject to a constant initial condition

$$
u(x, 0)=\lambda .
$$

An artificial perturbation parameter $\varepsilon=1$ is substituted into (33) as:

$$
F\left(u_{x x}, u_{t}, u, \varepsilon\right)=S+R=u_{t}+\varepsilon\left(-u_{x x}-u+u^{2}\right)=0
$$

where $S=u_{t}$ and $R=\varepsilon\left(-u_{x x}-u+u^{2}\right)$.

\section{PIA-1 solution:}

For the Eq. (35), we have

$$
a=b=\alpha=1 .
$$

By substituting (36) into Eq. (9), PIA-1 turns into

$$
\left(\left(u_{c}\right)_{n}\right)_{t}=\left(u_{n}\right)_{x x}+u_{n}-\left(u_{n}\right)_{t}-u_{n}^{2} .
$$


One may choose the initial function as:

$$
u_{0}=C_{0}=\lambda
$$

which satisfies the initial condition. Using the Eqs. (13), (37), (38) with initial condition, the iterations are reached as:

$$
\begin{gathered}
u_{1}=\lambda+\lambda t-\lambda^{2} t \\
u_{2}=\lambda+\lambda t-\lambda^{2} t-\frac{1}{6} t^{2}(-1+\lambda) \lambda\left(3-6 \lambda-2 t \lambda+2 t \lambda^{2}\right) \\
u_{3}=\lambda+\lambda t-\lambda^{2} t-\frac{1}{6} t^{2}(-1+\lambda) \lambda\left(3-6 \lambda-2 t \lambda+2 t \lambda^{2}\right) \\
-\frac{(-1+\lambda) \lambda}{1260}\left(\begin{array}{l}
210 t^{3}-840 t^{3} \lambda-420 t^{4} \lambda-63 t^{5} \lambda+840 t^{3} \lambda^{2}+1260 t^{4} \lambda^{2} \\
+483 t^{5} \lambda^{2}+70 t^{6} \lambda^{2}-840 t^{4} \lambda^{3}-840 t^{5} \lambda^{3}-280 t^{6} \lambda^{3}-20 t^{7} \lambda^{3} \\
+420 t^{5} \lambda^{4}+350 t^{6} \lambda^{4}+60 t^{7} \lambda^{4}-140 t^{6} \lambda^{5}-60 t^{7} \lambda^{5}+20 t^{7} \lambda^{6}
\end{array}\right)
\end{gathered}
$$

and so on. In [30] , the exact solution is given by

$$
u(x, t)=\frac{\lambda e^{t}}{1-\lambda+\lambda e^{t}} .
$$

It is worth mentioning that the coefficients of higher order terms of PIA-1 solutions deviate from the Taylor expansion of (42) after the second term $u_{1}$.

\section{PIA-2 solution:}

Proceeding as in PIA-1, Eq. (11) reduces to

$$
\left(\left(u_{c}\right)_{n}\right)_{t}+2 u_{n}\left(u_{c}\right)_{n}=\left(u_{n}\right)_{x x}+\left(u_{n}\right)-\left(u_{n}\right)_{t}-\left(u_{n}\right)^{2} .
$$

for the Eq. (35). Using the same starting function $u_{0}=C_{0}=\lambda$ and applying the initial condition gives

$$
u_{1}=\lambda-\frac{1}{2} \mathrm{e}^{-2 t \lambda}\left(-1+\mathrm{e}^{2 t \lambda}\right)(-1+\lambda)
$$

For the other iterations, the term $u_{n}$ in the left hand side of equation (43) can be approximated as $u_{0}=\lambda$ for simplicity. By considering this, we have

$$
\begin{gathered}
u_{2}=\lambda-\frac{1}{2} \mathrm{e}^{-2 t \lambda}\left(-1+\mathrm{e}^{2 t \lambda}\right)(-1+\lambda)-\frac{\mathrm{e}^{-4 t \lambda}(-1+\lambda)\left(1-2 \mathrm{e}^{2 t \lambda}+\mathrm{e}^{4 t \lambda}-\lambda+\mathrm{e}^{4 t \lambda} \lambda-4 \mathrm{e}^{2 t \lambda} t \lambda^{2}\right)}{8 \lambda} . \\
u_{3}=u_{2}+\frac{e^{-8 \lambda t}}{384 \lambda^{3}} \times\left[\begin{array}{l}
1-6 e^{2 t \lambda}+18 e^{4 t \lambda}-10 e^{6 t \lambda}-3 e^{8 t \lambda}-4 \lambda+3 e^{2 t \lambda} \lambda+36 e^{4 t \lambda} \lambda \\
-96 e^{4 t \lambda} t^{2} \lambda^{5}-35 e^{6 t \lambda} \lambda+24 e^{6 t \lambda} t \lambda+6 \lambda^{2}+27 e^{2 t \lambda} \lambda^{2}-84 e^{4 t \lambda} \lambda^{2}+ \\
21 e^{6 t \lambda} \lambda^{2}+30 e^{8 t \lambda} \lambda^{2}-12 e^{2 t \lambda} t \lambda^{2}+48 e^{4 t \lambda} t \lambda^{2}+24 e^{6 t \lambda} t \lambda^{2}- \\
120 e^{6 t \lambda} t^{2} \lambda^{5}+48 e^{4 t \lambda} t^{2} \lambda^{6}+120 e^{6 t \lambda} t^{2} \lambda^{6}+\cdots
\end{array}\right]
\end{gathered}
$$

and so on. One can proceed to reach higher iterations by using a symbolic computer program. This problem has been studied by several authors via different methods. Table 1 and Table 2 display the 3- terms approximation obtained by Adomian decomposition method (ADM) [29], variational iteration method (VIM) [31], homotopy perturbation method (HPM) [32], PIA-1 with 2-terms and 3 -terms approximation of PIA-2 for different $\lambda$ 's. It is clear that PIA-2 gives better results than other techniques even for $n=2$. and different values of $\lambda$.

Example 2: Consider the nonlinear Fisher's problem [29, 32]

$$
u_{t}=u_{x x}+6 u(1-u)
$$


Table 1: The approximate solutions of Fisher equation (33) using different methods for $\lambda=2$.

\begin{tabular}{|l|l|l|l|l|l|}
\hline $\mathrm{t}$ & ADM,VIM,HPM & PIA-1 & PIA-2,n=2 & PIA-2,n=3 & Exact Solution \\
\hline 0.1 & 1.82867 & 1.82604 & 1.82657 & 1.82622 & 1.82621 \\
0.2 & 1.70933 & 1.6909 & 1.69581 & 1.69327 & 1.69309 \\
0.3 & 1.634 & 1.57935 & 1.59677 & 1.58924 & 1.58833 \\
0.4 & 1.59467 & 1.48076 & 1.52199 & 1.5068 & 1.50412 \\
0.5 & 1.58333 & 1.3876 & 1.4659 & 1.44119 & 1.43527 \\
0.6 & 1.592 & 1.29459 & 1.42414 & 1.38902 & 1.37818 \\
0.7 & 1.61267 & 1.19822 & 1.3933 & 1.34773 & 1.3303 \\
0.8 & 1.63733 & 1.09665 & 1.37069 & 1.31526 & 1.28976 \\
0.9 & 1.658 & 0.989778 & 1.35422 & 1.28993 & 1.25515 \\
1. & 1.66667 & 0.879365 & 1.34228 & 1.27032 & 1.2254 \\
\hline
\end{tabular}

Table 2: The approximate solutions of Fisher equation (33) using different methods for $\lambda=4$.

\begin{tabular}{|l|l|l|l|l|l|}
\hline $\mathrm{t}$ & ADM,VIM,HPM & PIA-1 & PIA-2,n=2 & PIA-2,n=3 & Exact Solution \\
\hline 0.1 & 3.172 & 3.10241 & 3.11587 & 3.11188 & 3.11166 \\
0.2 & 2.896 & 2.50177 & 2.62569 & 2.59558 & 2.591 \\
0.3 & 2.884 & 1.96033 & 2.34994 & 2.2723 & 2.25029 \\
0.4 & 2.848 & 1.41888 & 2.19833 & 2.06834 & 2.01102 \\
0.5 & 2.5 & 0.953571 & 2.1172 & 1.9425 & 1.83452 \\
0.6 & 1.552 & 0.718907 & 2.07478 & 1.86721 & 1.69955 \\
0.7 & -0.284 & 0.710974 & 2.05303 & 1.82349 & 1.59347 \\
0.8 & -3.296 & 0.185132 & 2.04203 & 1.79877 & 1.50829 \\
0.9 & -7.772 & -3.43782 & 2.03654 & 1.7851 & 1.4387 \\
1. & -14. & -16.3429 & 2.03383 & 1.77768 & 1.38104 \\
\hline
\end{tabular}

subject to the initial condition

$$
u(x, 0)=\left(1+e^{x}\right)^{-2} .
$$

(47) can be written as

$$
F\left(u_{x x}, u_{t}, u, \varepsilon\right)=S+R=u_{t}+\varepsilon\left(-u_{x x}-6 u+6 u^{2}\right)=0
$$

where $R=\varepsilon\left(-u_{x x}-6 u+6 u^{2}\right)$ and $S=u_{t}$.

\section{PIA-1 solution:}

For the Eq. (47), we have

$$
a=\alpha=1, b=6 .
$$

By considering (50) and setting $\varepsilon=1$, Eq. (9) becomes

$$
\left(\left(u_{c}\right)_{n}\right)_{t}=\left(u_{n}\right)_{x x}+6 u_{n}-\left(u_{n}\right)_{t}-6 u_{n}^{2} .
$$

Selecting the Eq.(48) as a starting function and using the formula, the iterations in (13) are reached as:

$$
u_{0}=C_{0}=\left(1+\mathrm{e}^{x}\right)^{-2}
$$




$$
\begin{gathered}
u_{1}=\frac{1}{\left(1+\mathrm{e}^{x}\right)^{2}}+\frac{10 \mathrm{e}^{x} t}{\left(1+\mathrm{e}^{x}\right)^{3}} \\
u_{2}=\frac{1}{\left(1+\mathrm{e}^{x}\right)^{2}}+\frac{10 \mathrm{e}^{x} t}{\left(1+\mathrm{e}^{x}\right)^{3}}+\frac{25 \mathrm{e}^{x} t^{2}\left(-1+3 \mathrm{e}^{2 x}+2 \mathrm{e}^{3 x}-8 \mathrm{e}^{x} t\right)}{\left(1+\mathrm{e}^{x}\right)^{6}} \\
u_{3}=u_{2}+\frac{25 t^{3} e^{x}\left(-6 e^{x}-15 e^{2 x}+20 e^{3 x}+5\right)}{3\left(e^{x}+1\right)^{6}}-\frac{50 t^{4} e^{2 x}\left(5 e^{x}+52 e^{2 x}-17\right)}{\left(e^{x}+1\right)^{8}} \\
-\frac{150 t^{5} e^{2 x}\left(-47 e^{x}+20 e^{3 x}+5\right)}{\left(e^{x}+1\right)^{9}}+\frac{10000 t^{6} e^{3 x}\left(2 e^{x}-1\right)}{\left(e^{x}+1\right)^{10}}-\frac{240000 t^{7} e^{4 x}}{7\left(e^{x}+1\right)^{12}}
\end{gathered}
$$

\section{PIA-2 solution:}

In the light of previous example, one can construct PIA-2 as:

$$
\left(\left(u_{c}\right)_{n}\right)_{t}+12\left(u_{n}\right)\left(u_{c}\right)_{n}=\left(u_{n}\right)_{x x}+6\left(u_{n}\right)-\left(u_{n}\right)_{t}-6\left(u_{n}\right)^{2} .
$$

For the sake of simplicity and easier calculating, the term $u_{n}$ in the left hand side of equation (56) is taken as 0.25 which comes from

$$
u_{0}=\left(1+\mathrm{e}^{x}\right)^{-2}=0.25-0.25 x+0.0625 x^{2}+O[x]^{3} .
$$

Thus, we have

$$
\left(\left(u_{c}\right)_{n}\right)_{t}+3\left(u_{c}\right)_{n}=\left(u_{n}\right)_{x x}+6\left(u_{n}\right)-\left(u_{n}\right)_{t}-6\left(u_{n}\right)^{2}
$$

for (49). Using the equations (13), (52) and (58) with initial condition yields

$$
\left.\begin{array}{c}
u_{1}=\frac{1}{\left(1+\mathrm{e}^{x}\right)^{2}}-\frac{10 \mathrm{e}^{-3 t+x}\left(-1+\mathrm{e}^{3 t}\right)}{3\left(1+\mathrm{e}^{x}\right)^{3}} \\
u_{2}=u_{1}+\frac{10 \mathrm{e}^{-6 t+x}\left(\begin{array}{l}
-2 \mathrm{e}^{6 t}+20 \mathrm{e}^{x}-11 \mathrm{e}^{6 t+x}+24 \mathrm{e}^{6 t+2 x} \\
-13 \mathrm{e}^{3(t+x)}(1+3 t)-24 \mathrm{e}^{3 t+2 x}(1+3 t) \\
+\mathrm{e}^{3 t}(2+6 t)+\mathrm{e}^{3 t+x}(-9+93 t)+13 \mathrm{e}^{6 t+3 x}
\end{array}\right)}{9\left(1+\mathrm{e}^{x}\right)^{6}} \\
u_{3}=u_{2}+\frac{5 e^{-12 t}}{243\left(e^{x}+1\right)^{12}}\left(\begin{array}{l}
16000 e^{4 x}-72 e^{9 t+x}+72 e^{12 t+x}-840 e^{6 t+2 x}- \\
138 e^{9 t+2 x}+978 e^{12 t+2 x}+16320 e^{6 t+3 x}-24792 e^{9 t+3 x} \\
+8472 e^{12 t+3 x}-6000 e^{3 t+4 x}+79200 e^{6 t+4 x}-99808 e^{9 t+4 x} \\
+10608 e^{12 t+4 x}+\cdots
\end{array}\right.
\end{array}\right)
$$

and so on. The solution in a closed form by Wazwaz [29] given by

$$
u(x, t)=\left(1+e^{x-5 t}\right)^{-2} .
$$

The problem is already solved with ADM [29], HPM [32]. However, table (3) reveals that PIA-2 solutions gives more effective results than ADM [29], HPM [32] and PIA-1 solutions for $n=3$. Figure 1 also shows the accuracy of the fourth order PIA-2 solutions.

Example 3: Consider the nonlinear generalized Fisher's problem [29, 32]

$$
u_{t}=u_{x x}+u\left(1-u^{6}\right)
$$


Table 3: The four terms approximate solutions of Fisher equation (47) using different methods.

\begin{tabular}{|l|l|l|l|l|}
\hline$(x, t)$ & ADM and VIM & PIA-1 & PIA-2 & Exact Solution \\
\hline$(0.1,0.1)$ & 0.359069 & 0.358299 & 0.358301 & 0.358426 \\
$(0.2,0.2)$ & 0.485955 & 0.473909 & 0.474520 & 0.476064 \\
$(0.3,0.3)$ & 0.637904 & 0.578701 & 0.579214 & 0.590630 \\
$(0.4,0.4)$ & 0.831367 & 0.651047 & 1.521991 & 0.692254 \\
$(0.5,0.5)$ & 1.089006 & 0.668100 & 0.741226 & 0.775803 \\
$(0.6,0.6)$ & 1.435358 & 0.608198 & 0.801921 & 0.840572 \\
$(0.7,0.7)$ & 1.892066 & 0.453814 & 0.852762 & 0.888637 \\
$(0.8,0.8)$ & 2.473569 & 0.195085 & 0.896315 & 0.923202 \\
$(0.9,0.9)$ & 3.183966 & -0.166481 & 0.933343 & 0.947513 \\
\hline
\end{tabular}

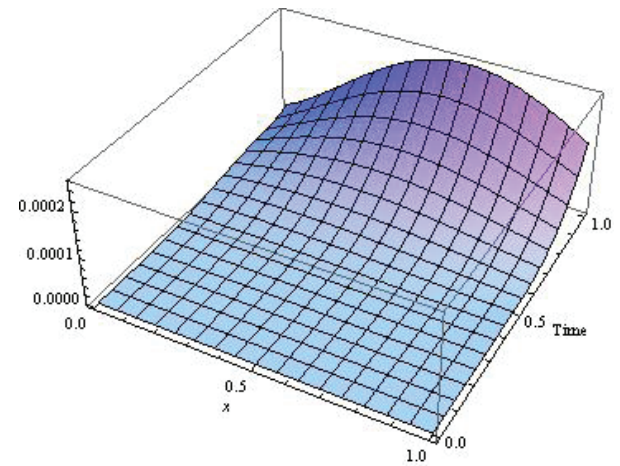

(a) Fourth order PIA-1 solution.

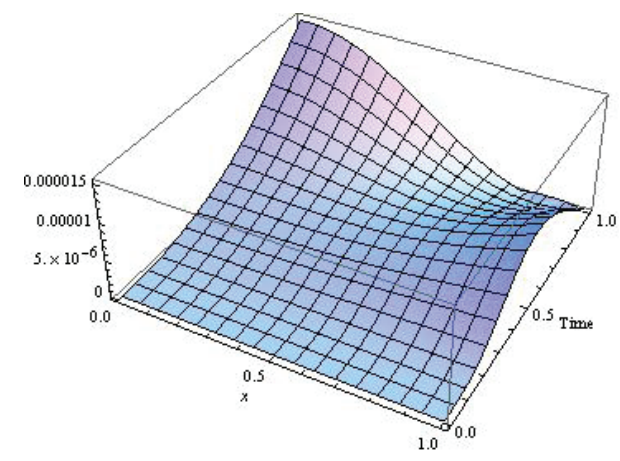

(b) Fourth order PIA-2 solution.

Figure 1: Absolute errors obtained by PIM for Example 2.

subject to the constant initial condition

$$
u(x, 0)=\left(1+e^{(3 x / 2)}\right)^{-1 / 3} .
$$

(63) is considered as

$$
F\left(u_{x x}, u_{t}, u, \varepsilon\right)=S+R=u_{t}+\varepsilon\left(-u_{x x}-u+u^{6}\right)=0
$$

where $R=\varepsilon\left(-u_{x x}-u+u^{6}\right), S=u_{t}$ and $a=b=1, \alpha=6$. Exact solution of this problem is given as [33]

$$
u(x, t)=\left(\frac{1}{2}-\frac{1}{2} \tanh \left[\frac{3}{4}\left(x-\frac{5 t}{2}\right)\right]\right)^{1 / 3} .
$$

\section{PIA-1 solution:}

For this problem, PIA-1 is obtained as:

$$
\left(\left(u_{c}\right)_{n}\right)_{t}=\left(u_{n}\right)_{x x}+\left(u_{n}\right)-\left(u_{n}\right)_{t}-\left(u_{n}\right)^{7} .
$$


With the aid of the equations (13),(64) and (67), we get

$$
\begin{gathered}
u_{0}=C_{0}=\left(1+e^{(3 x / 2)}\right)^{-1 / 3} \\
u_{1}=\left(1+e^{(3 x / 2)}\right)^{-1 / 3}+\frac{5 \mathrm{e}^{3 x / 2} t}{4\left(1+\mathrm{e}^{3 x / 2}\right)^{4 / 3}} \\
u_{2}=u_{1}+\frac{25 t^{2} e^{\frac{3 x}{2}}\left(\frac{1}{e^{\frac{3 x}{2}}+1}\right)^{28 / 3}}{131072}\left[\begin{array}{l}
-12288+16384 e^{\frac{21 x}{2}}+4096 e^{12 x}-3584 e^{3 x}(8+5 t)^{2}- \\
8192 e^{\frac{3 x}{2}}(10+7 t)-3584 e^{\frac{9 x}{2}}\left(96+160 t+100 t^{2}+25 t^{3}\right) \\
-2240 e^{6 x}\left(128+256 t+240 t^{2}+120 t^{3}+25 t^{4}\right)+\cdots
\end{array}\right]
\end{gathered}
$$

\section{PIA-2 solution:}

Proceeding as in the other examples, PIA-2 can be formed as:

$$
\left(\left(u_{c}\right)_{n}\right)_{t}+7\left(u_{n}\right)^{6}\left(u_{c}\right)_{n}=\left(u_{n}\right)_{x x}+\left(u_{n}\right)-\left(u_{n}\right)_{t}-\left(u_{n}\right)^{7}
$$

The term $u_{n}$ in the left hand side of (71) is taken as $\frac{1}{2^{1 / 3}}$ due to

$$
u_{0}=C_{0}=\left(1+e^{(3 x / 2)}\right)^{-1 / 3}=\frac{1}{2^{1 / 3}}-\frac{x}{4 \cdot 2^{1 / 3}}-\frac{x^{2}}{16 \cdot 2^{1 / 3}}+O[x]^{3} .
$$

Therefore, (71) turns into simplified form:

$$
\left(\left(u_{c}\right)_{n}\right)_{t}+(7 / 4)\left(u_{c}\right)_{n}=\left(u_{n}\right)_{x x}+\left(u_{n}\right)-\left(u_{n}\right)_{t}-\left(u_{n}\right)^{7} .
$$

After making the relevant calculations, the following results are obtained:

$$
\begin{aligned}
& u_{0}=C_{0}=\left(1+e^{(3 x / 2)}\right)^{-1 / 3} \\
& u_{1}=\left(1+e^{(3 x / 2)}\right)^{-1 / 3}-\frac{5}{7} e^{\frac{3 x}{2}}\left(-1+e^{-\frac{7 t}{4}}\right)\left(\frac{1}{1+e^{\frac{3 x}{2}}}\right)^{4 / 3}
\end{aligned}
$$

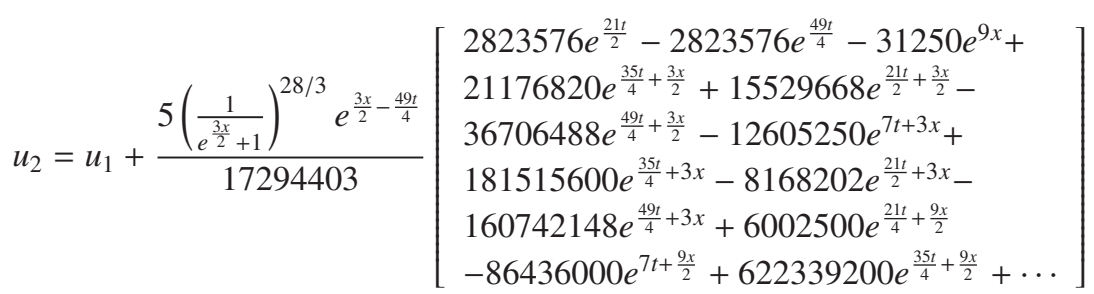

and so on. Table 4 shows a comparison between the four terms approximate solutions obtained by ADM [29], PIA-1 and PIA-2. Figure 2 represents the absolute errors of five terms PIM solutions.

\section{Discussion}

In this study, perturbation iteration method (PIM) is improved and new iteration algorithms are constructed to solve nonlinear partial differential equations (PDEs). These algorithms are employed specifically to nonlinear generalized Fisher's equations to get more effective and approximate solutions. The obtained solutions are compared with exact solutions as well as Adomian decomposition method (ADM), variational iteration method (VIM) and homotopy perturbation method (HPM). Approximate results have been also displayed in the tables and figures to justify the efficiency and accuracy of the proposed algorithms. Finally, we can say that this paper presents a detailed study of the newly developed perturbation iteration technique for PDEs and it will be encouraging for further studies. 
Table 4: The three terms approximate solutions of Fisher equation (63) by ADM and PIM.

\begin{tabular}{|l|l|l|l|l|}
\hline$(x, t)$ & ADM & PIA-1 & PIA-2 & Exact Solution \\
\hline$(0.1,0.1)$ & 0.822572 & 0.822003 & 0.822209 & 0.822296 \\
$(0.2,0.2)$ & 0.850714 & 0.845988 & 0.846902 & 0.848388 \\
$(0.3,0.3)$ & 0.879949 & 0.872917 & 0.872255 & 0.871810 \\
$(0.4,0.4)$ & 0.912240 & 0.907152 & 0.892304 & 0.892509 \\
$(0.5,0.5)$ & 0.949442 & 0.928362 & 0.919901 & 0.910540 \\
$(0.6,0.6)$ & 0.993089 & 0.898014 & 0.915520 & 0.926041 \\
$(0.7,0.7)$ & 1.044232 & 0.880825 & 0.908841 & 0.939211 \\
$(0.8,0.8)$ & 1.103356 & 0.901478 & 0.926074 & 0.950285 \\
$(0.9,0.9)$ & 1.170372 & 0.915556 & 0.928809 & 0.959515 \\
\hline
\end{tabular}

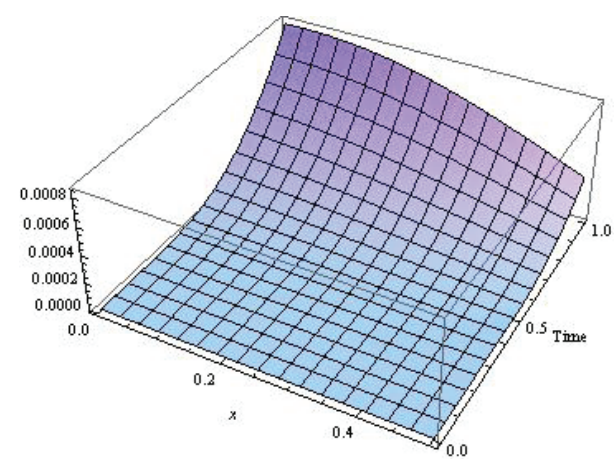

(a) Fifth order PIA-1 solution.

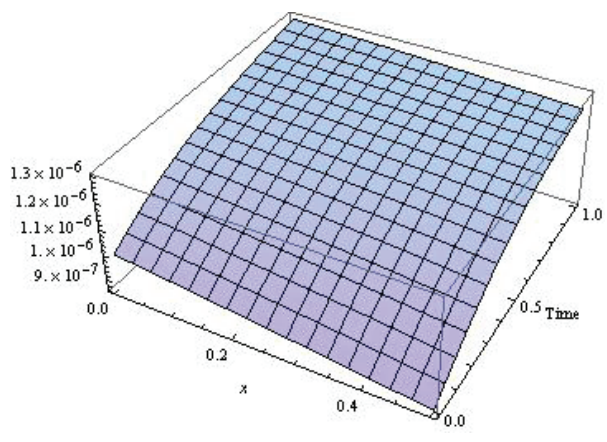

(b) Fifth order PIA-2 solution.

Figure 2: Absolute errors obtained by PIM for Example 3.

\section{Acknowledgement}

The authors would like to thank Manisa Celal Bayar University for their financial support.

\section{References}

[1] A Abbaoui, K., and Y. Cherruault. "Convergence of Adomian's method applied to nonlinear equations." Mathematical and Computer Modelling 20.9 (1994): 69-73.

[2] Evans, David, and Hasan Bulut. "A new approach to the gas dynamics equation: An application of the decomposition method." International journal of computer mathematics 79.7 (2002): 817 822.

[3] He, Ji-Huan. "Variational iteration method-a kind of non-linear analytical technique: some examples." International journal of non-linear mechanics 34.4 (1999): 699-708.

[4] Deniz, S., Bildik, N.,"Comparison of Adomian decomposition method and Taylor matrix method in solving different kinds of partial differential equations", International Journal of Modelling and Optimization, Vol.4(4): pp.292-298, 2014.

[5] Bildik, N., Deniz, S., "Comparison of solutions of systems of delay differential equations using Taylor collocation method, Lambert W function and variational iteration method." Scientia Iran- 
ica. Transaction D, Computer Science E Engineering and Electrical Engineering, Vol.22(3): pp.1052-1058, (2015).

[6] Deniz, S., "Optimal perturbation iteration method for solving nonlinear heat transfer equations." Journal of Heat Transfer-ASME, 139:37,074503-1, (2017).

[7] Bildik, N., Deniz, S., "Modification of perturbation-iteration method to solve different types of nonlinear differential equations", AIP Conf. Proc., 1798, 020027 (2017).

[8] Deniz, S., Bildik, N., "Applications of optimal perturbation iteration method for solving nonlinear differential equations", AIP Conf. Proc., 1798, 020046 (2017).

[9] Abbasbandy, S. "A new application of He's variational iteration method for quadratic Riccati differential equation by using Adomian's polynomials." Journal of Computational and Applied Mathematics 207.1 (2007): 59-63.

[10] Öziş, Turgut, and Deniz Ağırseven. "He's homotopy perturbation method for solving heat-like and wave-like equations with variable coefficients." Physics Letters A 372.38 (2008): 5944-5950.

[11] Turkyilmazoglu, M. "Convergence of the homotopy perturbation method." International Journal of Nonlinear Sciences and Numerical Simulation 12.1-8 (2011): 9-14.

[12] Abbasbandy, Saeid. "Numerical solutions of the integral equations: Homotopy perturbation method and Adomian's decomposition method." Applied Mathematics and Computation 173.1 (2006): 493-500.

[13] Liao, Shijun. "On the homotopy analysis method for nonlinear problems." Applied Mathematics and Computation 147.2 (2004): 499-513.

[14] Abbasbandy, S. "Homotopy analysis method for the Kawahara equation." Nonlinear Analysis: Real World Applications 11.1 (2010): 307-312.

[15] Inc, Mustafa. "Application of homotopy analysis method for fin efficiency of convective straight fins with temperature-dependent thermal conductivity." Mathematics and Computers in Simulation 79.2 (2008): 189-200.

[16] Shivanian, Elyas, and Saeid Abbasbandy. "Predictor homotopy analysis method: Two points second order boundary value problems." Nonlinear Analysis: Real World Applications 15 (2014): 89-99.

[17] Marinca, Vasile, and Nicolae Herişanu. "Application of optimal homotopy asymptotic method for solving nonlinear equations arising in heat transfer." International Communications in Heat and Mass Transfer 35.6 (2008): 710-715.

[18] Marinca, Vasile, et al. "An optimal homotopy asymptotic method applied to the steady flow of a fourth-grade fluid past a porous plate." Applied Mathematics Letters 22.2 (2009): 245-251.

[19] Herişanu, N., and Vasile Marinca. "Accurate analytical solutions to oscillators with discontinuities and fractional-power restoring force by means of the optimal homotopy asymptotic method." Computers $\mathcal{F}$ Mathematics with Applications 60.6 (2010): 1607-1615.

[20] Iqbal, S., and A. Javed. "Application of optimal homotopy asymptotic method for the analytic solution of singular Lane-Emden type equation." Applied Mathematics and Computation 217.19 (2011): 7753-7761.

[21] Gupta, A. K., and S. Saha Ray. "Comparison between homotopy perturbation method and optimal homotopy asymptotic method for the soliton solutions of Boussinesq-Burger equations." Computers $\mathcal{E}$ Fluids 103 (2014): 34-41.

[22] Bildik, Necdet, and Sinan Deniz. "A new efficient method for solving delay differential equations and a comparison with other methods." The European Physical Journal Plus 132.1 (2017): 51.

[23] Tang, S., and R. O. Weber. "Numerical study of Fisher's equation by a Petrov-Galerkin finite element method." The Journal of the Australian Mathematical Society. Series B. Applied Math- 
ematics 33.01 (1991): 27-38.

[24] Mavoungou, T., and Y. Cherruault. "Numerical study of Fisher's equation by Adomian's method." Mathematical and computer modelling 19.1 (1994): 89-95.

[25] Tyson, John J., and Pavel K. Brazhnik. "On traveling wave solutions of Fisher's equation in two spatial dimensions." SIAM Journal on Applied Mathematics 60.2 (2000): 371-391.

[26] Aksoy, Yiğit, and Mehmet Pakdemirli. "New perturbation-iteration solutions for Bratu-type equations." Computers $\mathcal{E}$ Mathematics with Applications 59.8 (2010): 2802-2808.

[27] Aksoy, Yigit, et al. "New perturbation-iteration solutions for nonlinear heat transfer equations." International Journal of Numerical Methods for Heat E Fluid Flow 22.7 (2012): 814828.

[28] Itoh, Shigeru. "Random fixed point theorems with an application to random differential equations in Banach spaces." Journal of Mathematical Analysis and Applications 67.2 (1979): 261-273.

[29] Wazwaz, Abdul-Majid, and Alice Gorguis. "An analytic study of Fisher's equation by using Adomian decomposition method." Applied Mathematics and Computation 154.3 (2004): 609620.

[30] Jone, D. S., and B. D. Sleeman. "Differential Equations and Mathematical Biology. 2003." Chapman\& Hall/CRC, New York.

[31] Matinfar, M., and M. Ghanbari. "Solving the Fisher's equation by means of variational iteration method." International Journal of Contemporary Mathematical Sciences 4.5-8 (2009): 343-348.

[32] Ağırseven, Deniz, and Turgut Öziş. "An analytical study for Fisher type equations by using homotopy perturbation method." Computers $\mathcal{E}$ Mathematics with Applications 60.3 (2010): 602609.

[33] Wang, X. Y. "Exact and explicit solitary wave solutions for the generalized Fisher equation." Physics Letters A 131.4-5 (1988): 277-279. 